\title{
Prediction of joint line remnant defect in Friction Stir Welding
}

\author{
Narges Dialami ${ }^{1}{ }^{*}$, Miguel Cervera ${ }^{1}$, Michele Chiumenti ${ }^{1}$ and Antonio Segatori ${ }^{2}$ \\ ${ }^{1}$ International Center for Numerical Methods in Engineering (CIMNE), Universidad \\ Politécnica de Cataluña, Campus Norte UPC, 08034 Barcelona, Spain; \\ Miguel.Cervera@upc.edu; michele@cimne.upc.edu \\ 2 Hydro, Innovation \& Technology - Europe Extruded Solutions, Finspang, Sweden; \\ Antonio.Segatori@ hydro.com (A.S.) \\ * Correspondence: narges@cimne.upc.edu; Tel.: +34 934016529
}

\begin{abstract}
\end{abstract}
This work studies computationally and experimentally the joint line remnant defects emerging in Friction Stir Welding (FSW) due to oxide layer propagation into the weld. A finite element-based model is used for the computational simulation. To follow the evolution of the oxide layer originally placed between the butted surfaces, a material tracing technique is incorporated in the numerical model. This approach allows tracking the position of the tracers representing oxide layer particles knowing the nodal velocities.

A robust and fast two-stage numerical strategy is adopted for the analysis of FSW process to solve the underlying thermo-mechanical problem. The first stage is a speedup stage solved on a fixed mesh that allows to quickly obtain the steady state. Oxide layer evolution is traced in the second stage where the rotation of the tool is modelled.

Experimentally, to produce a clearly dispersed oxide line in the weld, one of the workpieces is anodized while the other one is taken as extruded.

The computationally obtained oxide layer patterns are compared to those obtained experimentally using macrograph analysis of the joint cross section. The effect of the pin features and the process parameters on the final result is studied. The results show that with appropriate modelling of the material tracers in FSW, significant agreement can be attained between the computed and measured post-FSW oxide layer evolved results.

Keywords: FSW, Joint line remnant, Oxide line evolution, Computational modelling, Experimental validation

\section{INTRODUCTION}

Friction stir welding (FSW) is a joining technique characterized by the formation of a welding joint at temperatures underneath the melting point. One of the reasons of its popularity is the high quality of the resulting joint. As stated by Norton [1], conventional welding techniques result in the formation of multiple important defects, such as porosity, solidification cracking, liquation cracking and redistribution of solute. According to Yoshikawa [2] none of these are encountered in FSW welds.

However, due to the conditions of FSW joints occurrence such as inappropriate process parameters and insufficient heat input, some other types of defects appear $[3,4,5]$. In 
the butt welding, typical defects are voids and wormholes, lack of penetration and joint line remnant. It is possible to reduce the probability and, ideally, to completely eliminate defects via the choice of optimal FSW process parameters. Also, the quality of the workpiece edge preparation is a significant factor for making a defect-free joint. FSW guarantees the required strength of a joint and the absence of defects if the choice of the welding parameters is correct and the surfaces between the workpieces during the welding are clear and no oxide layers forms.

The initial oxide layer on the base material surface influences the resulting mechanical properties of FSW alloys. Joint line remnant defect (also referred to as "entrapped oxide defect") forms as a consequence of the creation of a nearly continuous oxide layer inside the weld nugget. In this case, if no metallurgical bond is formed along the defect, a zero-volume joint line emerges consequently. Joint line remnant generates from deficient deformation at the contacting surfaces of the two materials placed in tight contact to form a joint. Mishra and Mahoney [6] attributed it to either erroneous tool location with respect to the joint line, an absence of adequate mixing (too high rotational/advancing weld speeds), or cold processing conditions.

It can likewise be provoked by poor cleaning of the workpiece preceding welding. A joint line remnant shown by macrograph and at an increased magnification is depicted in figure 1 . The joint line remnant typically comprises an array of oxides that can be seen from the macrostructure even at very low magnifications.

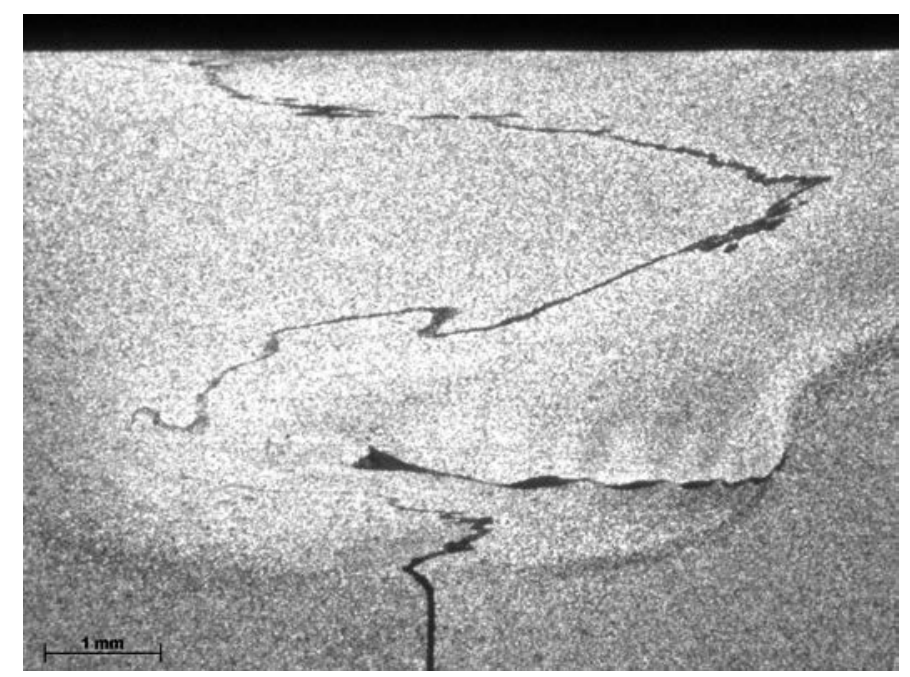

Figure 1. Joint line remnant imperfections in a FSW shown by macrographs.

Joint line remnant in FSW can be detected by both experimental and computational approaches. In recent studies, there has been a growing interest of the initial surface oxide layer behaviour during the weld and its effect on the creation of joint line remnant defect.

There are various experimental studies of the effect of oxide layer on the joint line remnant. Leonard and Lockyer [7] encounter a number of flaws in friction stir welds such as voids, joint line remnants and root flaws generated in an Al-Cu-Mn-Si-Mg alloy. The flaws are created using welding parameters outside the accepted range for producing flaw-free welds. Moreover, the distribution of oxide particles through the weld generates the joint line remnant. The welds are characterized using X-ray and ultrasonic non-destructive testing techniques and by metallographic sectioning.

Sato et al. [8] incorporate transmission electron microscope (TEM) to study the oxide layer behaviour along the remnant line. They find that the oxide particles are amorphous 
Al2O3. Due to the amorphous nature, the oxides are attributed to native oxide films on the aluminum surfaces preceding the FSW.

Most investigations demonstrate that the joint line remnant can be excluded by the appropriate choice of FSW process parameters of tool rotation, travel, and tilt. For instance, Kim et al. [9] conclude this for aluminum die casting alloy; Liu et al. [10] observe this behaviour for AA1050-H24 and AA6061-T6 aluminum alloys; as well as Crawford et al. [11] for AA 6061-T6 .

Nevertheless, as shown by Tarasov et al. [12] and Chen et al. [13], defects such as remnant joint lines due to oxide line evolution are poorly revealed by the existing nondestructive inspection experimental methods. These defects have an important impact as they may propagate cracks during the weld and therefore decrease the strength of the welded joint. The oxide layers are often conveyed to the weld from the edges of workpieces and are visible at the cross section of a joint. However, if the edges of the workpieces are clean before the welding process, these defects are hardly noticeable even during the metallographic analysis of the joint cross section.

Computationally, the joint line remnant defect due to oxide layer evolution during the weld has not been investigated in detail so far. However, numerical modelling has been used to study imperfections such as free surface changes (Fraser et al. [14]), void (Zhu et al. [15] and Al-Badour et al. [16]), tunnel-like (Atharifar et al. [17] and Shinoda and Kondo [18]) and wormhole (Zhu et al. [19]). Numerical methods enable a fast determination of the process parameters for experimental implementation. Numerical modelling of FSW has been extensively utilized as a mean to investigate the temperature field and to apprehend material flow. Further numerical examinations can help with better understanding the FSW mechanism and evading process condition for which imperfections may happen.

Material flow visualization can be a useful tool for numerical detection of defects originating from/generated by unclear surfaces. The shape of material flow in the vicinity of the tool for various process conditions can explain the evolution of such flaws.

Tutunchilar et al. [20] establish a 3D Lagrangian incremental finite element method for the simulation of friction stir processing. It permits prediction of the defect types, stir zone shape and material flow in the weld area. Material flow patterns are extracted following a selection of points in the Lagrangian domain. However they do not study joint line remnant defect detection.

De Vuyst el al. [21] analyse the flow of material around simplified tool profiles. A copper "marker insert technique" is used in their experiments. The copper sheets are embedded transversally or longitudinally to the weld interface. The results of the simulation correlate well with the experimental ones when copper markers are located transversely to the weld line. However, in the case of markers inserted along the weld centreline, the correlation needs to be improved.

Accurate prediction of the material flow depends on the robustness of the numerical model developed for the simulation of FSW. Dialami et al. [22, 23] use a material tracing method to model the material flow during the process from the original configuration to the final position. The material tracer trajectories are calculated incorporating the fourth order Runge-Kutta (RK4) time marching scheme. Material tracking is performed at the post-process level of the simulation step where the nodal velocities are known. These are used for the calculation of material tracer velocities and displacements. For the simulation step, following the approach proposed by Chiumenti et al. $[24,25]$, a staggered time-stepping algorithm is incorporated for the solution of the 
coupled thermo-mechanical problem. The thermal and mechanical sub-problems are solved sequentially.

Dialami et al. [26] propose a fast and robust FSW simulation strategy adopted here, designed specifically for the application to real industrial cases, where high precision should not be compromised by a high computational cost. The model is enriched with the enhanced friction model [27] that was shown to be responsible for providing not only the realistic thermal results but also mechanical results such as forces and torque.-It includes a microstructural model able to predict the average grain size and microhardness [28].

Despite the considerable amount of work dedicated to the numerical simulation of defects formation, the joint line remnant defects have not been previously analysed. The aim of the present paper is to evaluate the potential of numerical methods for detection of joint line remnant defect due to oxide layer propagation into the weld.

In this work, we strive to adapt the model developed by Dialami et al. for prediction of such defects. A numerical particle tracing technique is adopted for the oxide layer evolution as detecting the joint defect using common methods of radiography and nondestructive inspection is challenging. The material tracing technique incorporated in the fast and accurate FSW model helps to follow the trajectory of the oxide particles originally placed between the butting surfaces during the weld until the final position. The model is validated by comparing the obtained results with the experimental evidence provided from macrograph analysis of the joint cross section to assess the material behaviour and oxide line evolution within the entire process.

The structure of the paper is as follow: Section 2 briefly explains the numerical strategy used in this work. In section 3, the material tracing technique is described. In the next section, the oxide line distribution for two welding cases using circular pin and threaded pin is analysed. Finally, conclusions regarding the results of the experiments and the computational simulation are drawn for the effect of pin features and process parameters on the joint remnant defect.

\section{THE TWO-STAGE SIMULATION STRATEGY}

For the computational simulation of FSW, a domain containing the ThermoMechanically Affected Zone (TMAZ) is considered (figure 2). The tool is rotating around its axis with a constant velocity at a fixed location while the workpiece is moving with the advancing velocity opposite to the weld direction. A two-stage strategy is adopted, as proposed by Dialami et al. [26]. In both stages the coupled thermomechanical problem is solved. The first stage aims at reaching to the steady state speedily. This is achieved by accelerating the thermal diffusion in the thermal problem as the mechanical problem is quasi static. In this stage, the tool rotation is not considered and an Eulerian framework is adopted. Therefore, there is no periodic behaviour at his level. The second stage (periodic stage) considers the outcome of the first stage as the initial condition. In this stage, the tool rotation is considered.

This work focuses on the periodic stage of the simulation where the material flow due to the rotating and advancing movement of the tool are accounted for. In this stage, an apropos kinematic framework is adopted using a combination of Arbitrary Lagrangian Eulerian (ALE), Eulerian and Lagrangian schemes for different parts of the computational domain.

The use of an apropos kinematic framework in a FSW problem conveniently allows dealing with arbitrary pin profiles, applying the boundary conditions and also tracing 
the material flow. A Lagrangian formulation is used for the rigidly rotating tool. ALE and Eulerian formulations are used for the TMAZ and the remaining part of the analyzed domain, respectively. In ALE and Eulerian frameworks, the governing equations are written in terms of velocity (a fluid-like formulation). This choice of kinematic framework does not suffer from mesh distortion and does not require of remeshing. Details on the apropos kinematic framework are given by Dialami et al. [24].

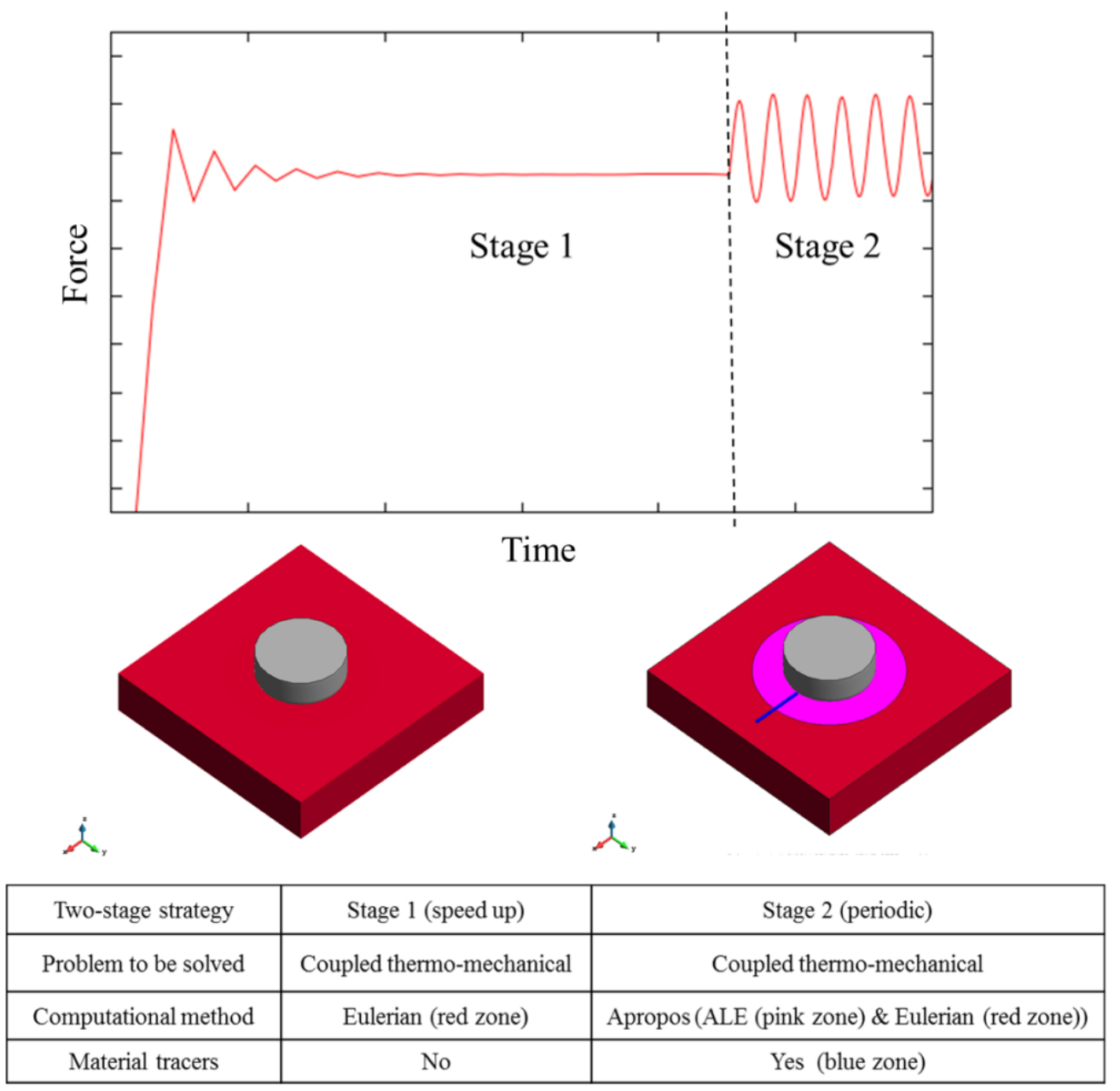

Figure 2. Two-stage strategy concept.

\section{MATERIAL TRACING}

In the ALE and Eulerian frameworks, the mesh discretizing the workpiece does not follow the material movement. Therefore, in order to find the location of the oxide particles during the weld an additional technique needs to be implemented. One option is to use the particle tracing technique which consists in finding the trajectories of the flowing material tracers during the weld from their initial position to their final location. To perform material tracing, a set of tracers representing the oxide layer is initially distributed in vertical plane at the interface between two butting surfaces. Figure 3 shows the domain used to perform the FSW simulation with material tracing. Note that material tracers in this case correspond to oxide particles. The tracing technique permits following the motion of each material tracer and finding its position at each time step of the simulation during the weld. 
The material position of a tracer in time, $\mathbf{X}(t)=\mathbf{X}$, is obtained by solving the following equation at each time step of the simulation:

$$
\frac{D \mathbf{X}}{D t}=\mathbf{V}(\mathbf{X}, t)
$$

where $\mathbf{V}(\mathbf{X}, t)$ is the material velocity of the tracer at the actual location (figure 4).

The solution of this equation restores the position of the tracers from already computed velocity of the material tracers as indicated by the time integration technology of the analysis. The fourth order Runge-Kutta (RK4) time marching formula is utilized as a part of this work. This strategy is picked because of its ability of integrating exactly a circular path (classical trajectory in FSW). The post-processing of this solution characterizes the profile path of the material tracers initially situated between the butting surfaces.

Equation (1) is integrated after each step of the simulation when the momentum and balance energy equations are solved. From the solution of the governing equations at each step, the velocity field is obtained.

A search algorithm is executed at each time step to discover the finite element surrounding each tracer. The velocity of the material tracers is obtained using the finite element nodal velocities and the corresponding interpolation (shape) functions. Once the velocities of the tracers are known, their position is obtained by integrating equation (1).

Additional information on the realized material tracing method is provided by Dialami et al. [22,23].

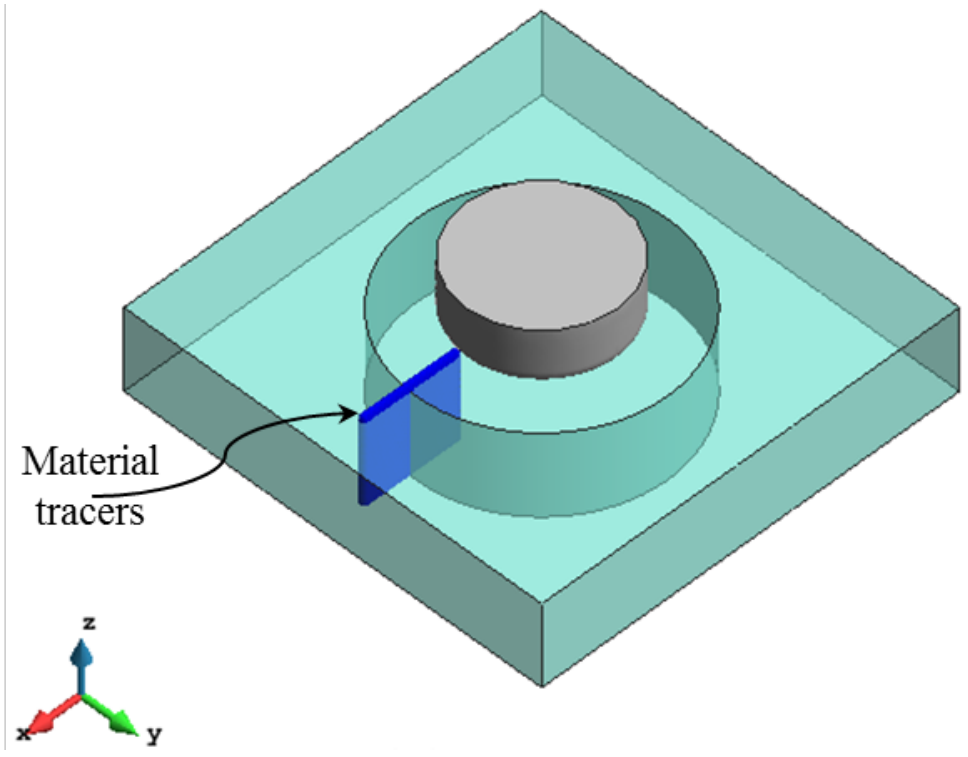

Figure 3. Area of FSW simulation with material tracer initial configuration. 


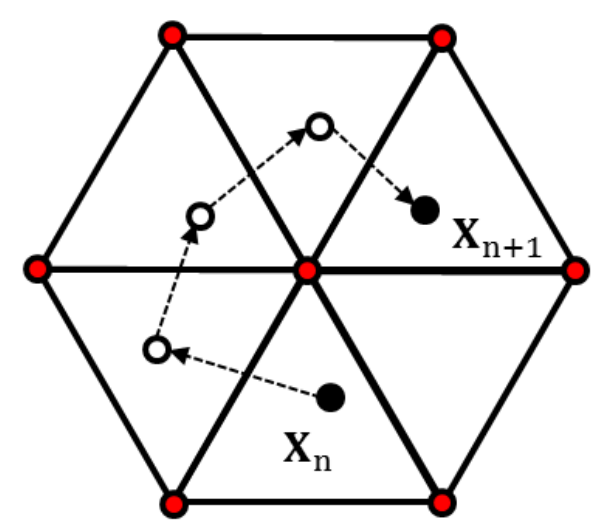

Figure 4. Evolution of a material tracer at 4 sub-time steps.

\section{EXPERIMENTAL SET UP}

In this section, the experimental set up used in this work is described. The clamping system used in all the performed tests is presented in figure 5. The two types of pin profiles, featureless and threaded cylindrical, are presented in figures 6 and 7. Apart from the presence of the thread in the latter one, both geometry dimensions are identical. The numerical geometry data for each tool profile is specified in the corresponding subsequent sections.

In the experiment, in order to visualize the oxide layer evolution, one of the workpieces is anodized while the other one is maintained as extruded. Oxide coating, which normally covers the surface of aluminium, is deliberately thickened by anodizing. The anodized workpiece is placed on the advancing side to be butt with the unanodized one. Afterwards, macrographs are taken from each weld. In this instance, a visible dispersed oxide line is produced in the weld, which is also of sufficient size to be detected by both radiography and ultrasonic inspection.

The post-FSW workpiece before macrograph analysis of the welded joint is depicted in figure 8 .

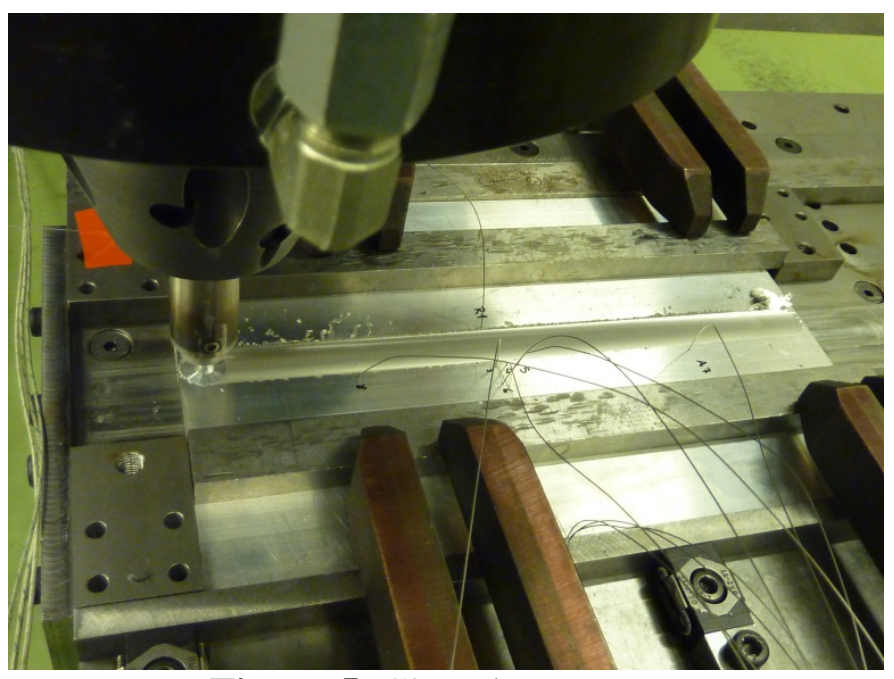

Figure 5. Clamping system. 


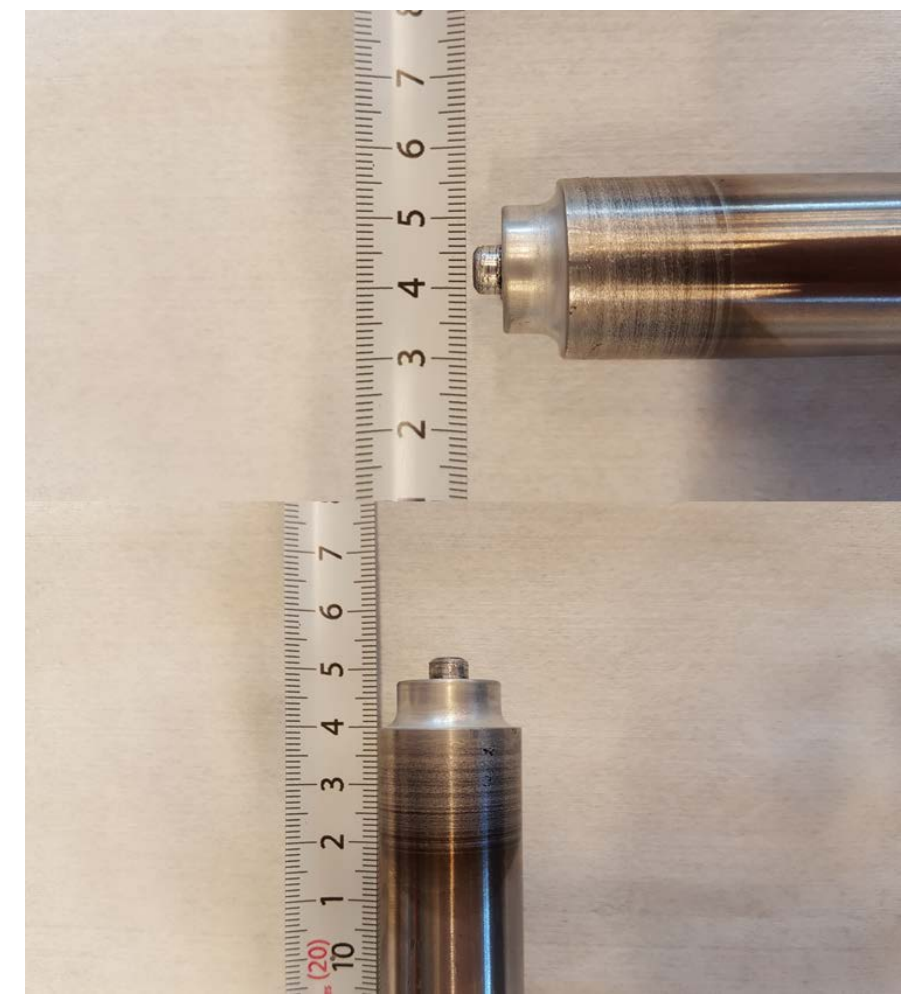

Figure 6. Featureless cylindrical pin. Geometry detail.

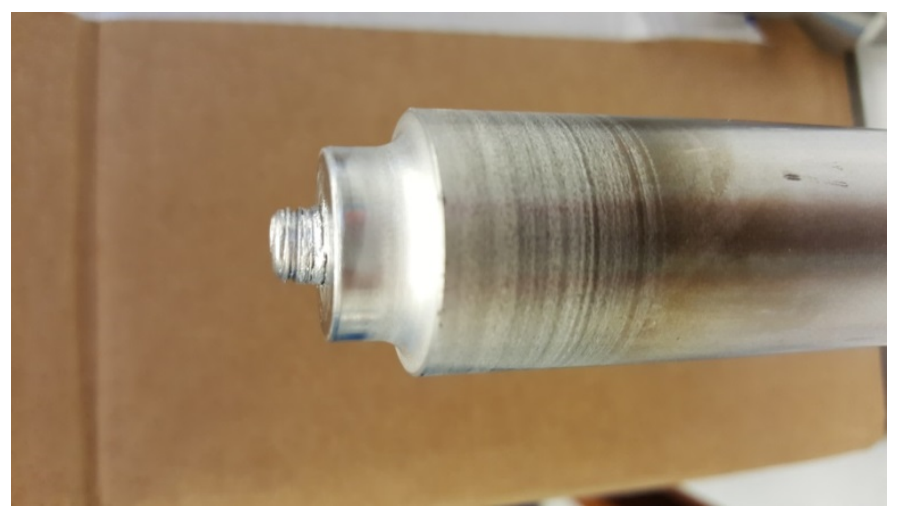

Figure 7. Circular threaded pin. Geometry detail. 


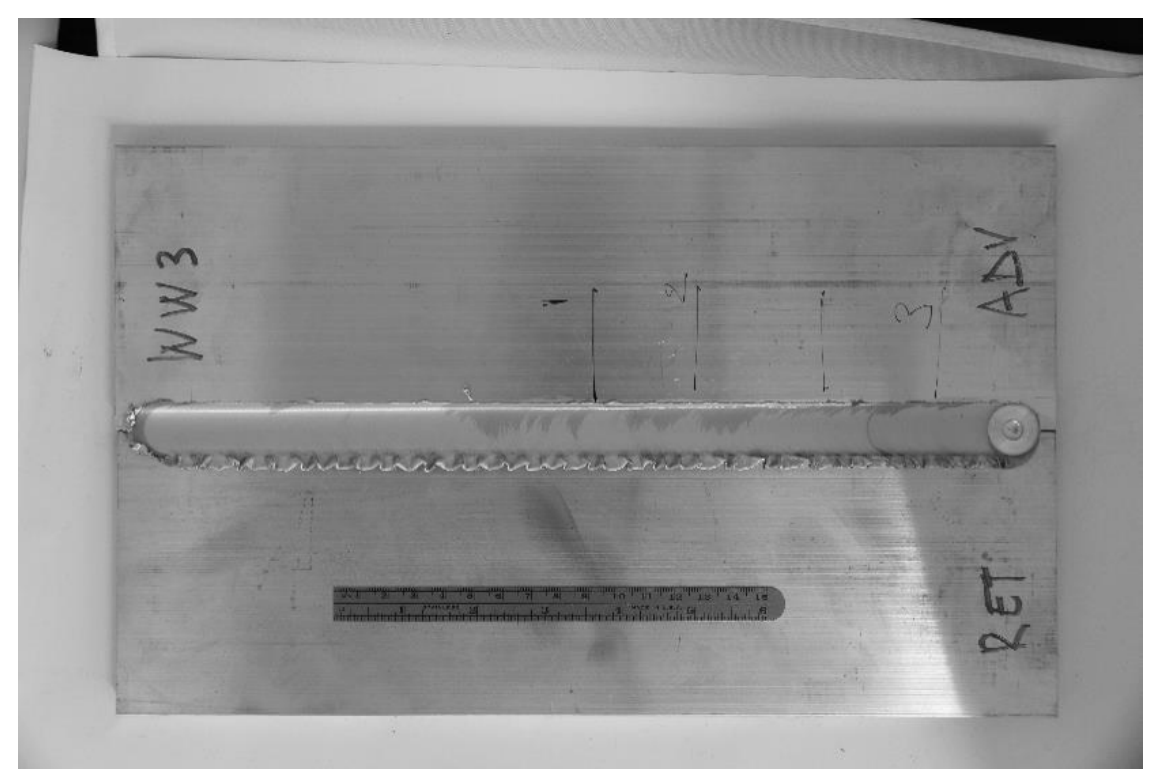

Figure 8. Circular threaded pin. Workpiece after welding.

\section{OXIDE LINE EVOLUTION}

In the following, three FSW cases are analysed to predict the joint line remnant defect due to oxide line evolution. These cases include different pin shapes and different process parameters:

- A featureless cylindrical pin,

- A threaded cylindrical pin and same process parameters and material properties as the first one,

- A threaded cylindrical pin with different process parameters from the second case.

The motivation for the selection of these three cases is to study the effect of the thread and the process parameters on the joint line remnant defect due to oxide line evolution during the weld.

\subsection{FEATURELESS CYLINDRICAL PIN}

In the present section, the numerical simulation of oxide line evolution in FSW process using a featureless cylindrical pin is performed.

A tool with a flat shoulder is used. The dimension of the tool is provided in figure 6 . The tool shoulder and pin diameters are 18 and $7 \mathrm{~mm}$, respectively. The tool pin height is $4 \mathrm{~mm}$. Zero tool tilt angle and no penetration of the shoulder into the workpiece is considered amid the entire welding process. The process parameters of tool advancing and rotating speeds are $400 \mathrm{~mm} /$ minute and $600 \mathrm{rpm}$, respectively. The material is aluminium alloy (Al6063-T6).

$\mathrm{X}$-axis is considered to be the tool advancing direction. Heat generation by both friction and plastic dissipation is taken into account. $70 \%$ of the rate of the plastic work is assumed to be dissipated as heat.

Friction is considered at the contact surfaces between pin/workpiece and shoulder/workpiece [27]. Material properties are temperature dependent. The best fitting of the experimental results in terms of temperature evolution as well as for the forces 
and torque calibration is obtained at the first stage of the simulation and is presented by Dialami et al. [27].

The computational mesh of 70,000 nodes and 400,000 tetrahedral elements is used in this simulation. A set of 30,000 material tracers representing the oxide layer is uniformly distributed in the domain of $0.2 \times 11 \times 10 \mathrm{~mm}^{3}$. They are placed vertically before the tool at the initial configuration. Figure 9 presents the mesh resolution at the tool and workpiece. In order to better specify the geometry detail and to accurately capture the steep temperature gradient in the TMAZ, a higher mesh resolution is utilized in the vicinity of the tool.

At the second stage, material tracers move from their initial location along the welding direction (negative $\mathrm{X}$-axis). They move to the rear side guided by the welding tool. After all the material tracers pass through the rotating tool, the transversal and vertical coordinates of the tracers become constant. Then, a transversal cut a'b' (yz plane) on the final steady state position (figure 10) of the tracers is made. This observation plane serves for analysing the oxide distribution in the joint area after welding and comparing it with the oxide line position in a transversal section of the experimental test.

The obtained pattern from the transversal cut $a^{\prime} b^{\prime}$ (figure 11) is overlapped on top of the experimental macrograph to facilitate the comparison (figure 12). RS and AS represent the retreating and advancing sides, respectively. Good agreement between the numerical result and experimental evidence is found.

The material tracers are distributed following the orientation of the flow of the stirred material. Variations observed in the distribution of the tracers in the depth direction (from the top of the nugget to the bottom) correspond to the differences in the dominant heat generation and material transport mechanisms. The oxide layer distribution forms a characteristic curved shape limiting the weld nugget on the RS, as confirmed by Dickerson et al. [29]. Material in the vicinity of the top surface is extended towards the AS because of the shoulder frictional force; in spite of material movement near the bottom (that is from AS to RS) due to the movement of the pin tool.

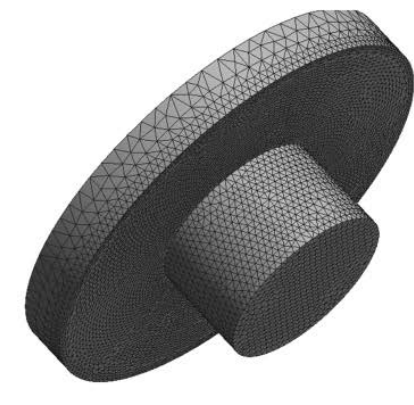

a)

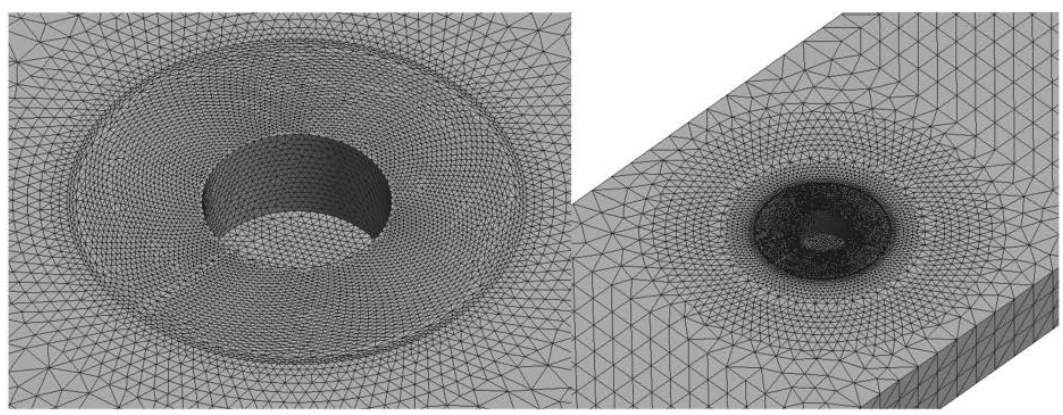

b) c)

Figure 9. Featureless cylindrical pin. Mesh discretization a) tool b) workpiece - stir zone c) workpiece. 


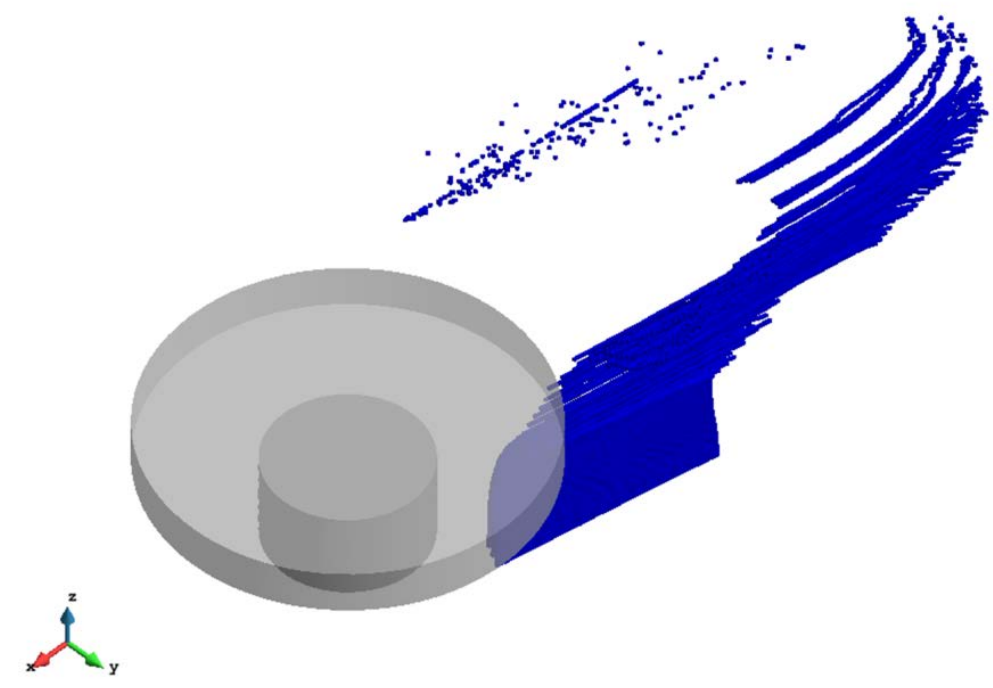

Figure 10. Featureless cylindrical pin. Final location of the material tracers posterior to crossing the tool.

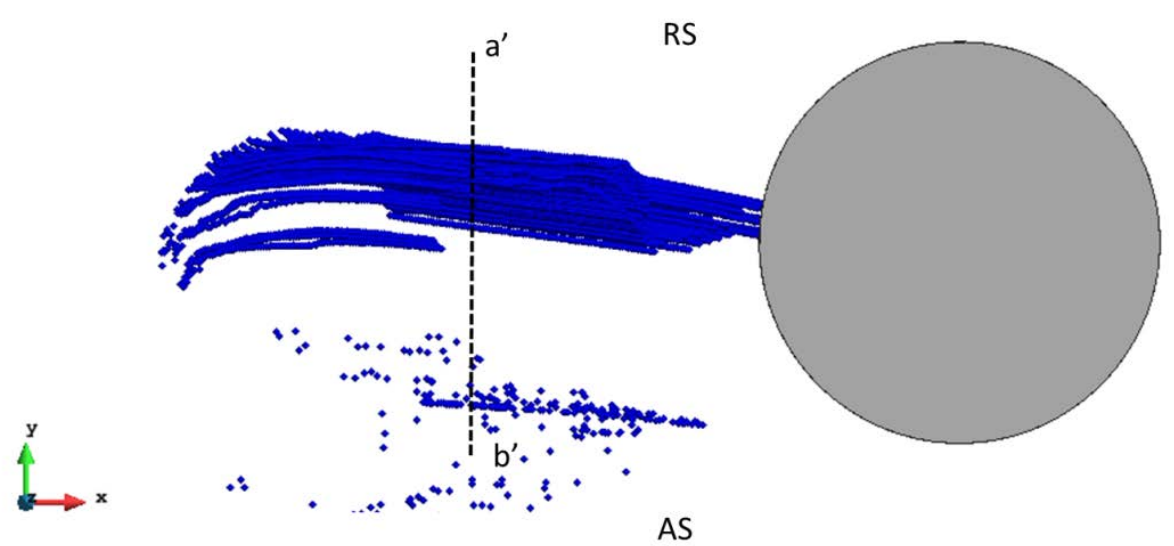

Figure 11. Featureless cylindrical pin. Oxide line distribution after steady state. 


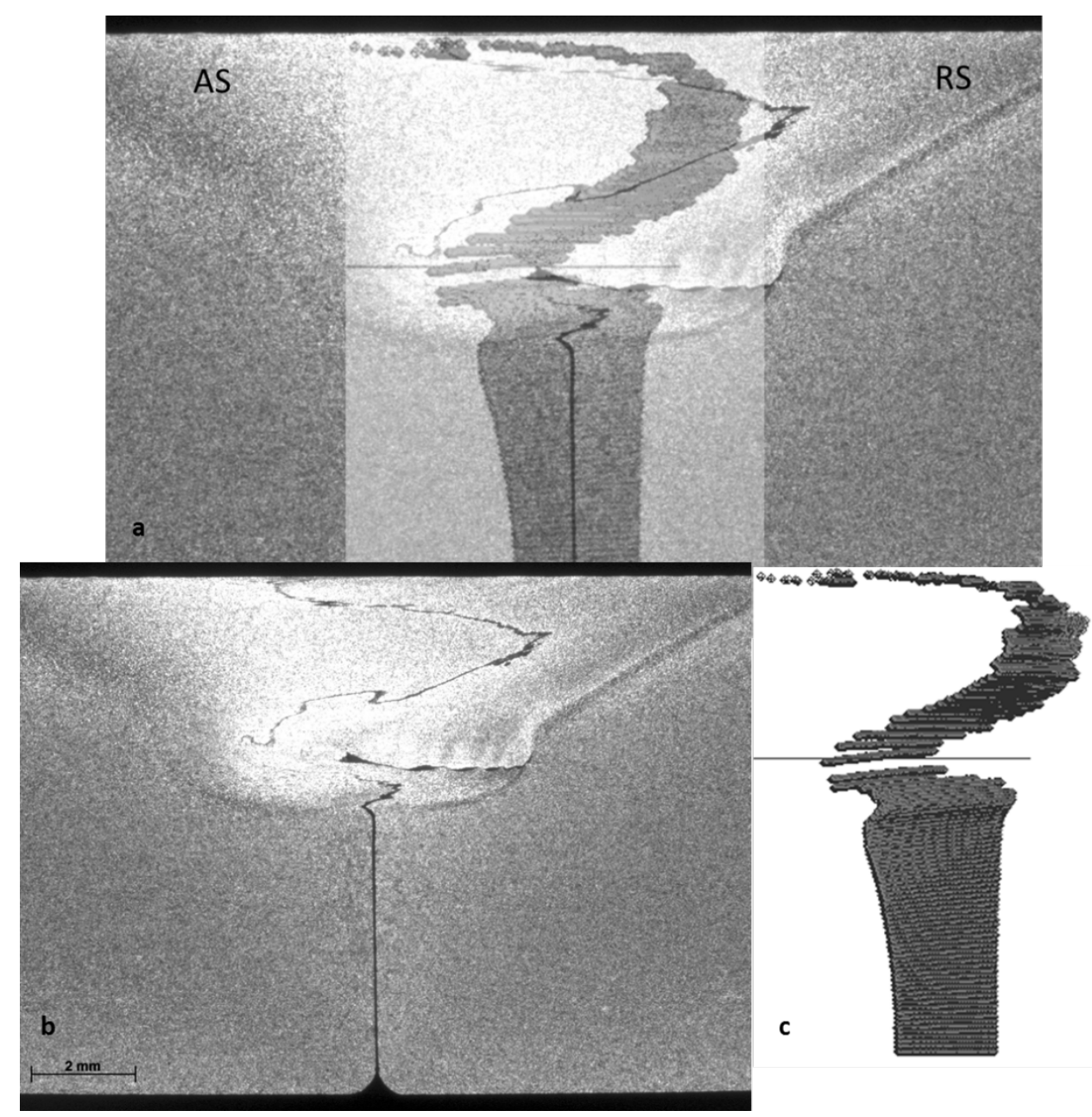

Figure 12. Featureless cylindrical pin. a) Comparison of simulation and experiment in terms of oxide line distribution (featureless cylindrical pin) b) experimental pattern c) simulation pattern.

\subsection{CIRCULAR THREADED PIN}

In the current section, the numerical simulation of oxide line evolution in FSW process using a threaded pin tool is performed as threads have a significant effect on material mixing and reducing the defects production [30].

The sizes of the relevant parts of the tool are as follows: The tool shoulder diameter $=$ $18 \mathrm{~mm}$, the tool pin average diameter $=7 \mathrm{~mm}$ and the tool pin height $=4 \mathrm{~mm}$ (figure 7). The process parameters of tool advancing and rotating speeds are $400 \mathrm{~mm} /$ minute and $600 \mathrm{rpm}$, respectively. The material is aluminium alloy (Al6063-T6). The material and process parameters are identical to the previous case of the featureless cylindrical pin. This is done in order to study the effect of threads on the final pattern of the evolved oxide line.

A local model of $50 \times 50 \times 10 \mathrm{~mm}^{3}$ is taken into account in the simulation. $\mathrm{X}$-axis is considered to be the tool advancing direction.

The computational mesh of 70,000 nodes and 400,000 tetrahedral elements is used in this simulation. A set of 30,000 material tracers representing the oxide layer is uniformly distributed in the domain of $0.2 \times 11 \times 10 \mathrm{~mm}^{3}$. Figure 13 shows the mesh 
resolution at the tool and workpiece. The mesh resolution in this test and the previous one (figures 9 and 13) is very similar from the point of view of distribution, density, element size and even number of elements.

Friction is considered at the contact surfaces between pin/workpiece and shoulder/workpiece. The parameters are chosen such that the best fitting of the experimental results in terms of temperature evolution as well as for the forces and torque calibration is obtained from the first stage of the analysis. These thermomechanical results are presented in reference [31].

At the initial stage, steady state temperature profile is studied. Previously mentioned advancing and rotating speeds are considered. The result of the thermo-mechanical numerical simulation done using a fixed-grid (Eulerian) model is compared against experimental results of Dialami et al. [31]. Various mechanical values (such as transversal, vertical and longitudinal forces as well as the torque) computed at the first stage are also compared against the experimental data.

The final steady state location of the material tracers is shown in figure 14 posterior to crossing the FSW tool. This configuration is attained after 60 cycles of the periodic phase of the thermo-mechanical simulation starting from the original state depicted in figure 3. Figure 8 displays the actual workpiece after performing the welding in the experiment.

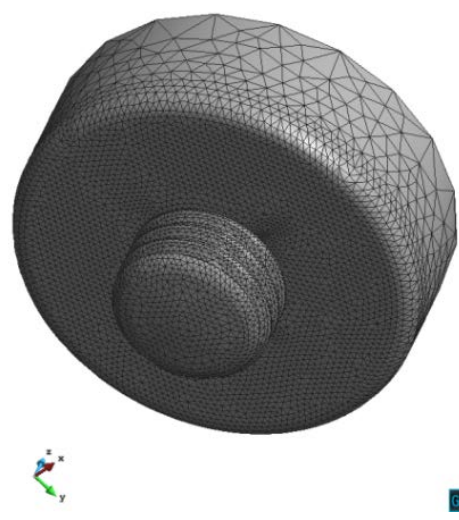

a)

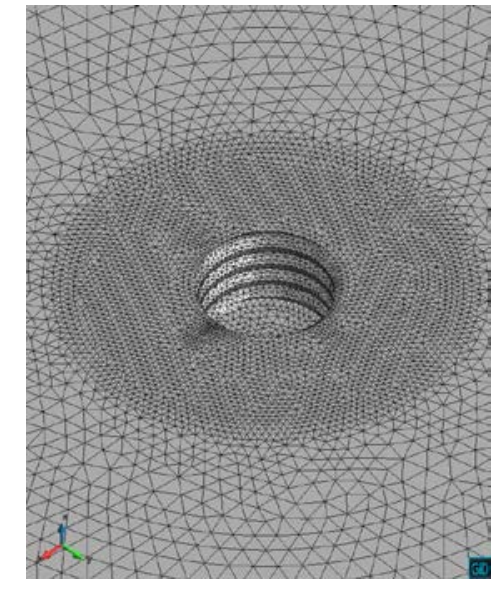

b)

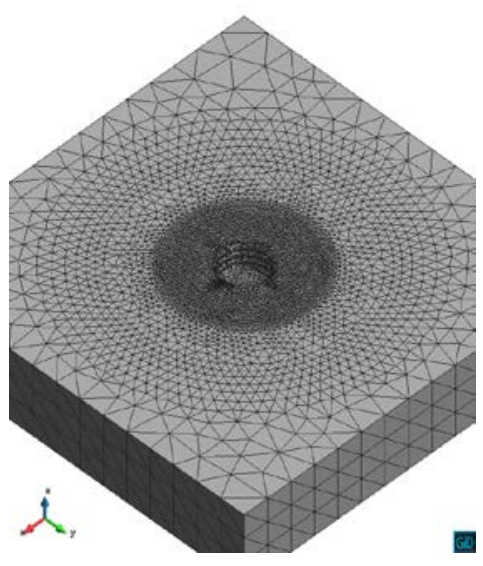

c)

Figure 13. Circular threaded pin. Mesh discretization a) tool b) workpiece - stir zone c) workpiece. 


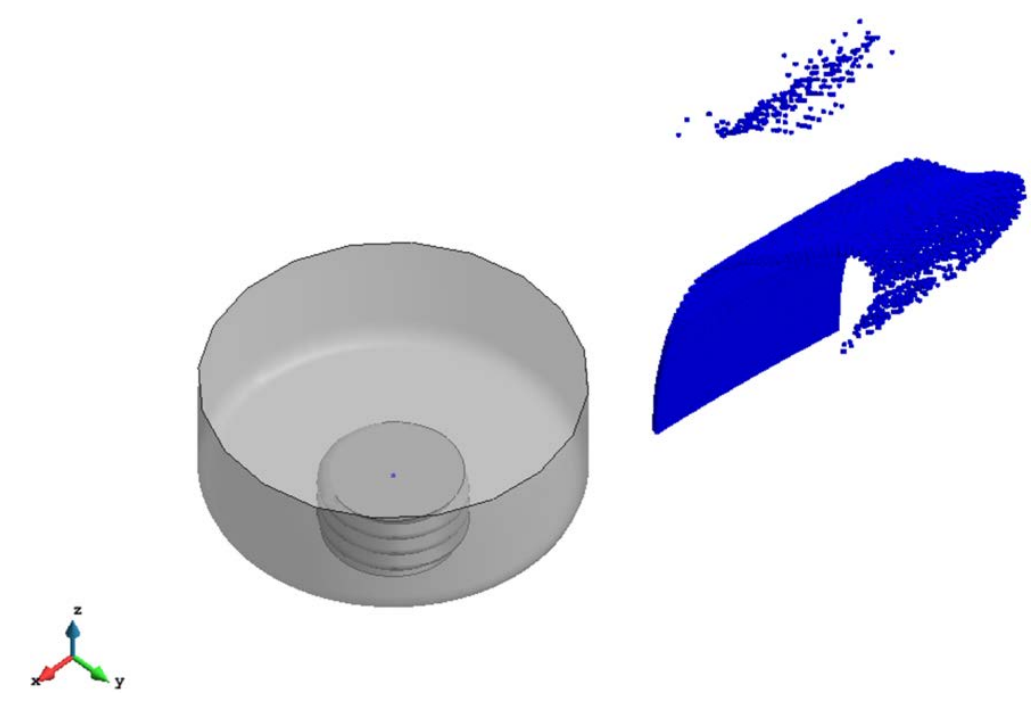

Figure 14. Circular threaded pin (advancing and rotating speeds are $400 \mathrm{~mm} / \mathrm{minute}$ and $600 \mathrm{rpm}$, respectively.). Oxide line distribution after steady state.

The objective of performing the second stage is to follow the oxide layer using tracers during the weld. At this step, the material follows the rotation of the tool. When all the material tracers have passed through the tool, a transversal cut a'b' on the final position of the tracers is made to be compared with the oxide line position in a transversal section obtained from the experiment (figure 15).

The result from the transversal cut a'b' is superimposed over the experimental result to facilitate the comparison (figure 16). Good agreement between the numerical result using a threaded pin and experimental evidence is found.

The curving path originating from the oxide layer remnants is shown. The ultimate location of the original oxidised joining layer transported by the material flow displays a curving path on the cross section. This is confirmed both experimentally and numerically. An oxide layer is trapped at the welding interface, and its ultimate location is captured from macrograph (in the experiment) and the material tracing technique (in the simulation).

The stirring movement of the oxide layer due to the thread effect is well reproduced in the computational model.

Comparing figures 12 and 16, the differences between the featureless and threaded pin under the same welding conditions become evident. Joint line remnant is clearly discernible in the cross section of the stir zone because of unclear surfaces of workpiece at initial state with remnant oxide layers, which is not disrupted as a result of inadequate deformation during stirring. It is revealed both experimentally and numerically that horizontal movements of the oxide layer take place due to the movement of the tool and effect of the shoulder. For the threaded pin profile, shoulder influence decreases with depth in form of a reduced deflection of oxide line. The underlying oxide layer is maneuvered with the flow of the material from RS to the rotating district close to the base of the pin. While for the featureless pin a C-shaped curved line is presented, oscillation in the place and more dispersion is seen in the case of the threaded pin as a result of the threads effect. 


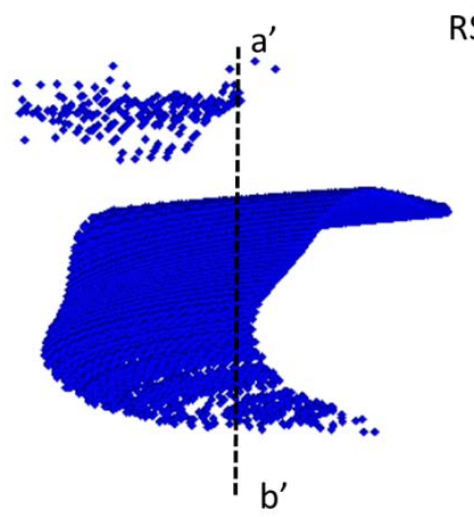

RS

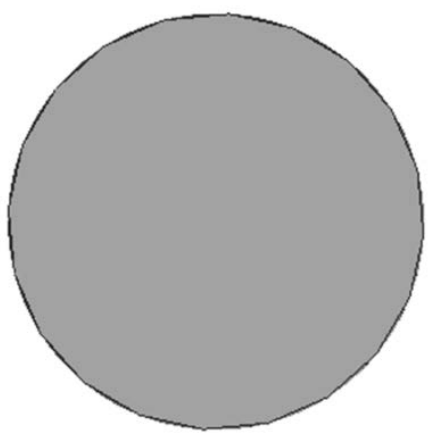

AS

Figure 15. Circular threaded pin (advancing and rotating speeds are $400 \mathrm{~mm} /$ minute and $600 \mathrm{rpm}$, respectively.). The transversal cut on the material tracers.

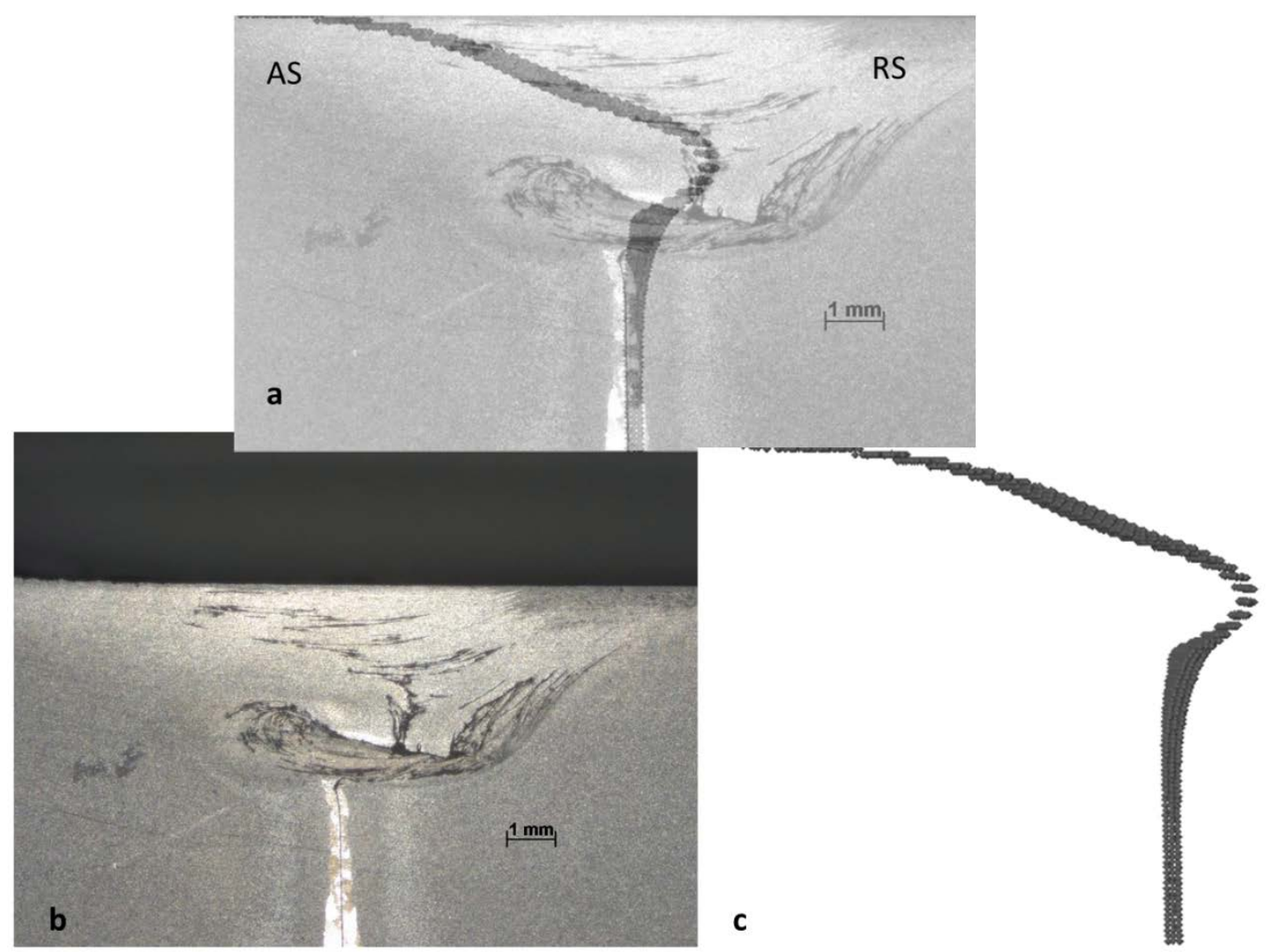

Figure 16. Circular threaded pin (advancing and rotating speeds are $400 \mathrm{~mm} /$ minute and 600 rpm, respectively.). a) Comparison of simulation and experiment in terms of oxide line distribution b) experimental pattern c) simulation pattern.

Experimental and numerical tests are also performed for a different set of process parameters: advancing speed $=600 \mathrm{~mm} /$ minute and rotating speed $=400 \mathrm{rpm}$. This is done in order to investigate how the process parameters affect the joint line remnant defect. A transversal cut (figure 17) is made on the final steady state position (figure 18) 
of the tracers to create an observation plane. The obtained result after superimposing the numerical and experimental visualization is shown in figure 19. Great match between numerical and experimental outcomes is observed also in this case, with the simulation capturing the S-shape of the remnant joint line.

Comparing these two sets of process parameters, both experimentally and numerically, significant differences are detected. In the first threaded case (figure 16), oxide material tends to move to the AS while in the second threaded case oxide material change the direction close to the top surface and evolves from AS to RS. This difference is due to the higher rotational and lower advancing velocities of the first case than the second one.
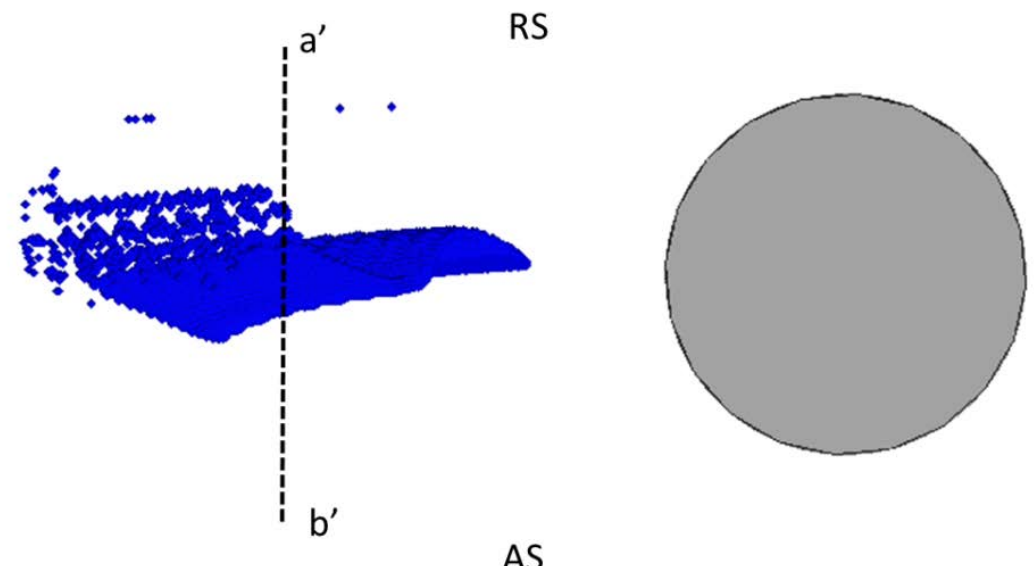

AS

Figure 17. Circular threaded pin (advancing and rotating speeds are $600 \mathrm{~mm} /$ minute and $400 \mathrm{rpm}$, respectively.). The transversal cut on the material tracers.
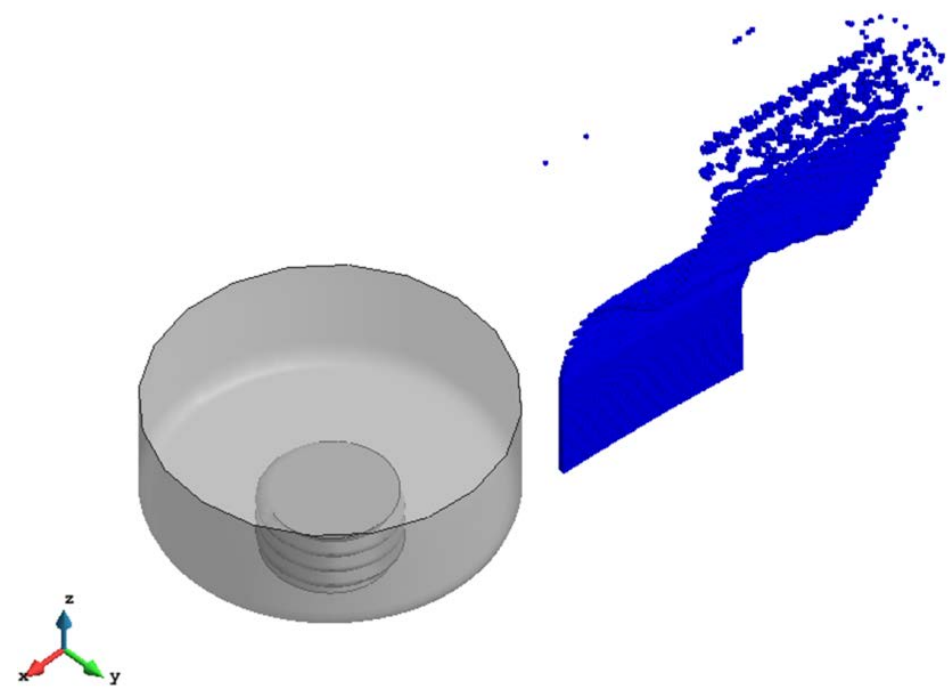

Figure 18. Circular threaded pin (advancing and rotating speeds are $600 \mathrm{~mm} / \mathrm{minute}$ and $400 \mathrm{rpm}$, respectively.). Oxide line distribution after steady state. 

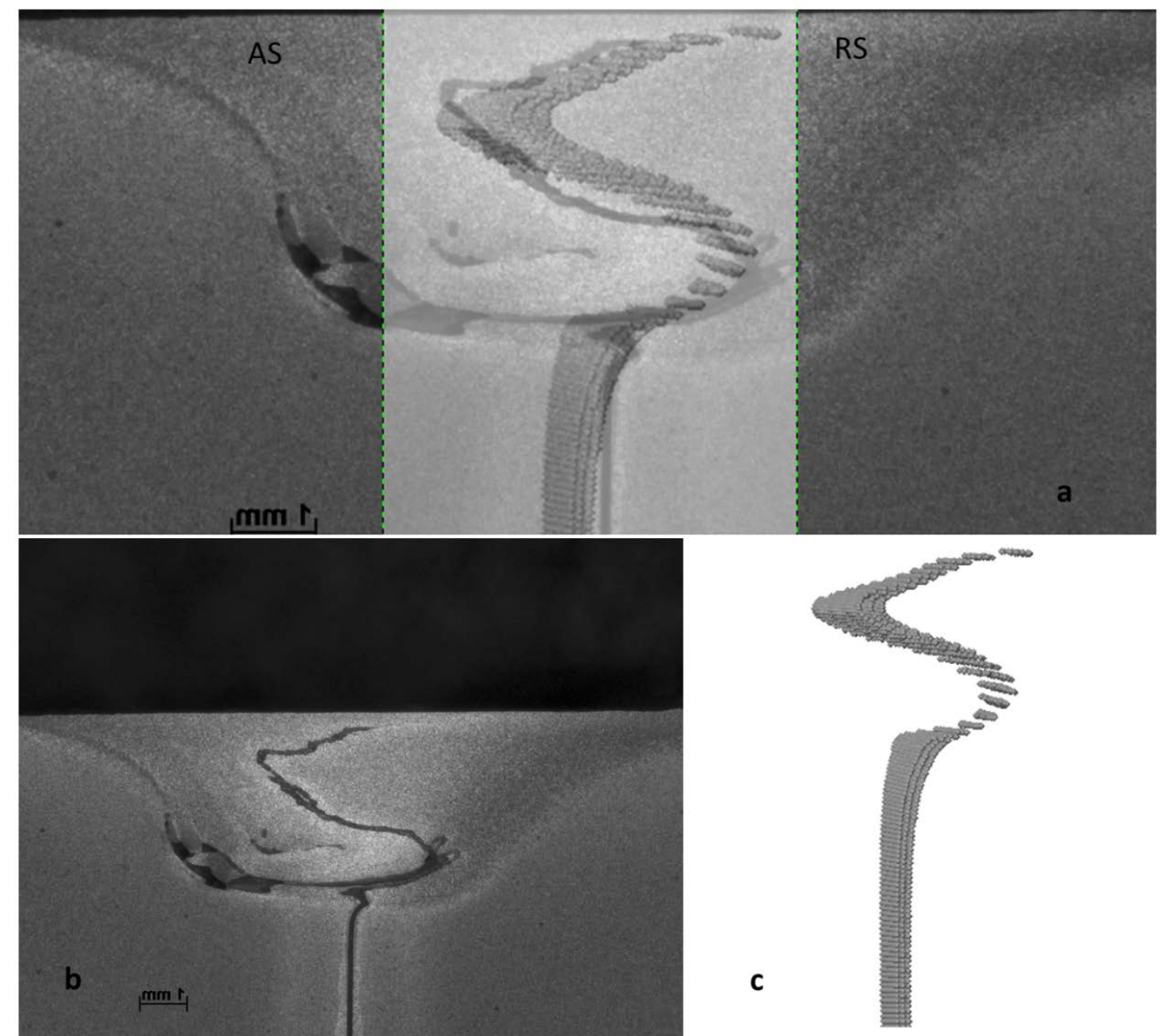

Figure 19. Circular threaded pin (advancing and rotating speeds are $600 \mathrm{~mm} / \mathrm{minute}$ and $400 \mathrm{rpm}$, respectively.). Oxide line comparison between numerical and experimental results. 


\section{SUMMARY AND CONCLUSIONS}

In this work, the results of a numerical simulation of remnant join line defect due to oxide layer evolution are presented for the first time. Featureless and threaded cylindrical tool pins are analysed.

A particle tracing technique is executed to follow the oxide particles during the weld to detect weld remnant joint defect.

The results are presented on a transversal cut at steady state when all the tracers pass through the tool and are compared with the experimental evidence. Material close to the top surface is stretched toward the AS because of the shoulder effect. In case of featureless pin, a C-shaped pattern appears different from the case with threaded pin revealing the effect of threads on the final oxide line pattern. In the case of a threaded pin, two sets of process parameters are studied and difference between these cases are observed, both numerically and experimentally. Due to the lower rotational and higher advancing velocities of the second threaded case than the first one, oxide material alters close to the top surface and drives from AS to RS.

The agreement between the numerical tracer trend and experimental results is encouraging in all cases. 


\section{REFERENCES}

[1] Norton F.H. (1929) The creep of steel at hight temperature. Mc Graw Hill, New York, USA.

[2] Yoshikawa, K. (2003) A joining criterion for lap joining of dissimilar metal materials of aluminum and stainless steel by friction stir. The 4th International Symposium on Friction Stir Welding, Park City, Utah, USA.

[3] Krishnan, K. (2002). On the formation of onion rings in friction stir welds. Materials Science and Engineering A, 327 (2), 246-251.

[4] Li B., Shen Y., Hu W. (2011) The study on defects in aluminum 2219-T6 thick butt friction stir welds with the application of multiple non-destructive testing methods, Materials and Design 32: 2073-2084.

[5] Grujicic, M., et al. (2010). Modeling of AA5083 material-microstructure evolution during butt friction stir welding. Journal of Materials Engineering and Performance, 19(5), 672-684.

[6] Mishra R., Mahoney M. (2007) Friction Stir Welding and Processing, Materials Park, OH,2007.

[7] Leonard A., Lockyer S. A. (2003) Flaws in Friction Stir Welding, 4th International Symposium on Friction Stir Welding, Park City, Utah, USA, 14-16 May.

[8] Sato Y.S., Yamashita F., Sugiura Y., Park S.H.C, Kokawa H. (2004) FIBassisted TEM study of an oxide array in the root of a friction stir welded aluminum alloy, Scripta Mater. 50: 365-369.

[9] Kim Y.G., Fujii H., Tsumura T., Komazaki T., Nakata K. (2006) Three defect types in friction stir welding of aluminum die casting alloy, Mater. Sci. Eng. A 415: 250-254.

[10] Liu H.J., Fujii H,, Maeda M., Nogi K. (2004) Tensile fracture location characterization of friction stir welded joints of different aluminum alloys, J. Mater. Sci. Technol. 20:103-105.

[11] Crawford R., Cook G.E., Strauss A.M., Hartman D.A., Stremler M.A. (2006) Experimental defect analysis and force prediction simulation of high weld pitch friction stir welding, Sci Technol Weld Joining 11: 657-665.

[12] Tarasov, S.Yu., Rubtsov, V.E., and Kolubaev, E.A. (2014) Radiographic detection of defects in friction stir welding on aluminum alloy AMg5M, AIP Conf. Proc., v. 1623, 631-634. doi:10.1063/1.4899024.

[13] Chen, HB., Yan, K., Lin, T., Chen, S-B., Jiang, C-Y., and Zhao, Y. (2006) The investigation of typical welding defects for 5456 aluminum alloy friction stir welds, Mater. Sci. Eng., A 433( 1-2): 64-69.

[14] Fraser K., St-Georges L. and Kiss L. I. (2016) A Mesh-Free SolidMechanics Approach for Simulating the Friction Stir-Welding Process, Joining Technologies SP - Ch. 3, InTech publisher http://dx.doi.org/10.5772/64159

[15] Zhu Z., Wang M., Zhang H. and Wu Z. (2017) A Finite Element Model to Simulate Defect Formation during Friction Stir Welding, Metals - Open Access Metallurgy Journal 7(7):256 DOI: 10.3390/met7070256

[16] Al-Badour F., Merah N., Shuaib A., Bazoune A. (2013) Coupled Eulerian Lagrangian finite element modeling of friction stir welding processes, Journal of Materials Processing Technology 213: 1433- 1439 
[17] Atharifar H, Lin DC, Kovacevic R; (2007) Studying tunnel-like defect in friction stir welding process using computational fluid dynamics: Mat. Sci. Tech., Detroit MI.

[18] Shinoda T, Kondo Y; (1997), Friction stir welding of aluminum plate: Welding Int., 11(3):179-184.

[19] Zhu Y., Chen G., Chen Q., Zhang G., Shi Q. (2016) Simulation of material plastic flow driven by non-uniform friction force during friction stir welding and related defect prediction, Materials and Design 108: 400-410.

[20] Tutunchilar S., Haghpanahi M., Besharati Givi M.K., Asadi P., Bahemmat P. (2012) Simulation of material flow in friction stir processing of a cast Al-Si alloy, Materials and Design 40: 415-426

[21] De Vuyst T., D’Alvise L., Robineau A., Goussain J.C. (2006), Simulation of the material flow around a friction stir welding tool, Proceedings of the 6th International Symposium on Friction Stir Welding (6ISFSW), SaintSauveur, Quebec, Canada, October 10-13

[22] Dialami N., Chiumenti M., Cervera M., Agelet de Saracibar C. and Ponthot J.-P. Material Flow Visualization in Friction Stir Welding via Particle Tracing (2013) International Journal of Metal Forming, 1-15.

[23] Dialami N., Chiumenti M., Cervera M., Agelet de Saracibar C. and Ponthot J.-P., Numerical simulation and visualization of material flow in friction stir welding via particle tracing (2014) Numerical Simulations of Coupled Problems in Engineering,157-169, Springer International Publishing.

[24] Dialami, N., Chiumenti, M., Cervera, M. and Agelet de Saracibar, C. (2013) An apropos kinematic framework for the numerical modeling of friction stir welding. Computers and Structures 117:48-57.

[25] Chiumenti, M., Cervera, M., Agelet de Saracibar, C. and Dialami, N. (2012) Numerical modeling of friction stir welding processes. Computer Methods in Applied Mechanics and Engineering, 254:353-369.

[26] Dialami N., Chiumenti M., Cervera M. and Agelet de Saracibar C., A fast and accurate two-stage strategy to evaluate the effect of the pin tool profile on metal flow, torque and forces during friction stir welding (2017) International Journal of Mechanical Sciences, 122, 215-227.

[27] Dialami N., Chiumenti M., Cervera M., Segatori A. and Osikowicz W., Enhanced friction model for Friction Stir Welding (FSW) analysis: simulation and experimental validation, (2017) International Journal of Mechanical Sciences, 133: 55-567.

[28] Dialami N., Cervera M. and Chiumenti M. (2018) Numerical modelling of microstructure evolution in Friction Stir Welding (FSW), Metals 8(3):183

[29] Dickerson T. L., Shercliff H. R. and Schmidt H. (2003) validation of friction stir welding process models, Proc. 4th Int. Symp. on 'Friction stir welding', Park City, UT, USA, May, TWI.

[30] Reza-E-Rabby Md., Reynolds A. P. (2014) Effect of Tool Pin Thread Forms on Friction Stir Weldability of Different Aluminum Alloys, Procedia Engineering 90: 637-642. 
[31] Dialami, N.; Cervera, M.; Chiumenti, M.; Segatori, A.; Osikowicz, W. (2017) Experimental validation of a FSW model with an enhanced friction model: Application to a threaded cylindrical pin tool. Metals 7: 491. 